\title{
On Avoiding-Minimizing Burst Collisions in Optical Burst-Switched Networks without Wavelength Conversion
}

\author{
Joan Triay ${ }^{*}$, Student Member, IEEE, Jordi Perelló ${ }^{\sharp}$, Cristina Cervelló-Pastor", Salvatore Spadaro ${ }^{\#}$ \\ * Department of Telematics Engineering, Universitat Politècnica de Catalunya (UPC) \\ Esteve Terradas, 7, 08860, Castelldefels, Spain \\ \# Advanced Broadband Communications Center (CCABA), Universitat Politècnica de Catalunya (UPC) \\ Jordi Girona 1-3, 08034, Barcelona, Spain \\ e-mail: joan.triay@entel.upc.edu,perello@ac.upc.edu
}

\begin{abstract}
In this paper, a new MAC protocol for OBS networks is introduced, described and evaluated. Into operation, DAOBS avoids burst collisions in the core of the network by controlling the way OBS nodes access the optical channel. Assuming no wavelength conversion capabilities in the network, DAOBS is assessed and compared to standard deflection routing through simulations. From the results, DAOBS offers zero collisions at low-tomedium network loads without excessively deteriorating the burst end-to-end delay. This makes DAOBS particularly useful either in network scenarios where the increased costs related to wavelength converters arise as prohibitive, or as a mid-term OBS network solution until wavelength conversion becomes sufficiently mature.
\end{abstract}

Keywords: optical burst switching (OBS), contention resolution, contention avoidance, MAC.

\section{INTRODUCTION}

Optical communication networks are expected to change radically in the near-term future. The advances experienced by new optical enabling technologies permits to foresee that, from the edge to the core of the wired network, optical networking will impose its superiority and advantages on providing huge raw bandwidth at impressive cost-bit rates. Nonetheless, due to the initial release and the narrower scope of diffusion and commercialization, these upcoming technologies may remain immature and especially expensive at the very beginning. This imposes on the research community to also engineer cost-efficient alternatives to cope with the ever-increasing bandwidth demands of new Internet applications.

Optical Burst Switching (OBS) [1] has been proposed as a practical paradigm for the future optical Internet using enabling optical technologies. The main goal of OBS is to enhance the performance of the current Optical Circuit Switching (OCS) technologies by exploiting the statistical multiplexing of bursts, and at the same time, alleviating the implementation complexities related to Optical Packet Switching (OPS) [2]. In OBS, packets are assembled into large bursts that later, after an offset time used by the Burst Control Packet (BCP) to reserve the resources along the path, are transmitted and switched all-optically.

Since optical burst-switched networks provide a connectionless transport, there exists the possibility that bursts may contend at intermediate nodes. Contention occurs if multiple bursts from different input ports try to leave the OBS switch through the same output port at the same time on the same wavelength. In view of this, effective contention resolution is critical in OBS networks to restrict losses to a reasonable low level. Wavelength conversion (WC), Fiber Delay Lines (FDL) and Deflection Routing (DR) are some of the techniques proposed to address this problem. Most of these techniques are reactive, that is, they are applied once the contention occurs.

In particular, DR is a reactive space-based domain contention resolution scheme [3]. Here, the contention is resolved sending the contending burst to a different output port of the switch and, consequently, on a different route towards the destination. Comparing the efficiency of different contention resolution strategies, DR alone provides only limited performance improvements. As a matter of fact, DR is typically implemented together with other contention resolution schemes, such as WC (in full, partial or sparse configurations), so that the global OBS network performance is enhanced.

In contrast, proactive contention resolution schemes try to prevent the network entering into a congestion state, hence avoiding the contentions to occur inside the network. This group involves techniques such as traffic routing, burst assembly or access-admission control. In this paper, we present a new MAC protocol for OBS that offers high burst delivery rates using current optical enabling technologies. The protocol Distributed Access for OBS (DAOBS) can be classified as an access-admission control scheme. This scheme is especially useful in scenarios where one of the main constraints is to reduce the CAPEX costs of using other more expensive optical equipment such as wavelength converters. Alternatively, it might also be used as a mid-term solution in the deployment of OBS networks, while WC or other more expensive/complex techniques are not fully available.

The remainder of the paper is organized as follows. A short description of the new protocol for OBS is given in section 2. Section 3 develops a comparison between DAOBS and other types of contention resolution, such as WC or DR, in order to evaluate the performance of the protocol. Finally, section 4 summarizes the main conclusions of the paper. 


\section{DISTRIBUTED ACCESS IN OPTICAL BURST SWITCHING}

Next we introduce the Distributed Access for Optical Burst Switching, or simply DAOBS. This MAC protocol for high-speed optical burst-switched networks is based on the IEEE 802.6 Distributed Queue Dual Bus (DQDB) Metropolitan Area Network (MAN) standard [4].

In the field of distributed medium access protocols, the main candidates are empty-slot protocols. These protocols switch fixed-length frames synchronously. The contention to access the medium is resolved by inserting new frames only when there are free slots on a given channel.

One of the main advantages of DAOBS is that, as bursts are only transmitted when free resources exist, burst contentions are avoided in the network. However, due to the own operation of the protocol, and the control packet propagation delays, a slight extra access delay is added, which in most situations does not represent a great issue compared to other contention resolution schemes such as DR.

\subsection{Basic operation of DAOBS and its implementation on mesh topologies}

In this section we briefly describe the original operation of the protocol that can be found in [5]. Later, we will illustrate some of the enhancements required to use the protocol on a mesh topology. For better comprehension, we exemplify its use along a light-path or optical bus (see Fig. 1). In such a case, we consider two unidirectional control channels, the downstream or forward channel, from the head of the bus (HoB) to the tail of the bus (ToB), and the upstream or reverse channel, from ToB to HoB. Both channels use the same path, one in each direction, and they can be in-fiber (using a specific wavelength), or out-of-fiber. A DAOBS entity is identified by its input port + output port + wavelength, and it is composed by (see Fig. 1):

- Two counters: a request counter (RQ_C) and a count-down counter (CD_C).

- A local queue (LQ) in which bursts waiting to access the optical bus/tree are temporarily stored.

- A distributed queue (DQ). A one-position queue that stores the burst while the node is in the active state.

The set of nodes' DQ of a specific bus/tree entity conform the so-called virtual distributed queue.

The protocol operates as follows. Each node can be in any of the following three states: idle, in case it has nothing to transmit on this optical bus; waiting, meaning that it has something to transmit but it has not made a successful request yet; or active, in case that the node has made a request and is waiting a free slot to transmit the corresponding burst. While a node is idle, it keeps track of the requests generated by the downstream stations with RQ_C. This is done by monitoring the request bit (REQ) of each Request Control Packet (RCP) travelling on the reverse channel, and the busy bit (BUSY) in the header of each BCP travelling on the forward channel.

When the node is in the waiting state, it must wait for a free $\mathrm{RCP}(\mathrm{REQ}=0)$. Just at that moment, the node becomes active by placing its burst in the virtual distributed queue (DQ). This process implies the dumping of the RQ_C value to the CD_C and the setting of REQ $=1$ to announce upstream nodes the request for a free slot. The CD_C is a measure of the outstanding requests queued for transmission by downstream nodes before the current node gets its turn to transmit. When the node is active it monitors the value of the BUSY bits, and for each free forwarded $\mathrm{BCP}(\mathrm{BUSY}=0)$, the $\mathrm{CD}_{-} \mathrm{C}$ is decrement by 1 . When the $\mathrm{CD}$ - reaches 0 , the node prepares itself to transmit using the next available free slot on the forward channel.

In [5], the basic piece of network to enable the use of DAOBS was the optical bus. This bus topology is now extended to a tree-based wavelength topology (see Fig. 2), which allows using DAOBS over more complex networks such as fully or partially meshed network topologies. Besides, a tree topology guarantees that a network node cannot receive a burst from two input ports to the same output port, avoiding in this way burst collisions. It shall be mentioned, however, that the rest of the nodes contained in the tree have also the right to transmit to other child nodes in the tree, and therefore there may still exist collisions when these other nodes are multiplexing their bursts on the optical channel. DAOBS is especially useful for avoiding this type of losses.

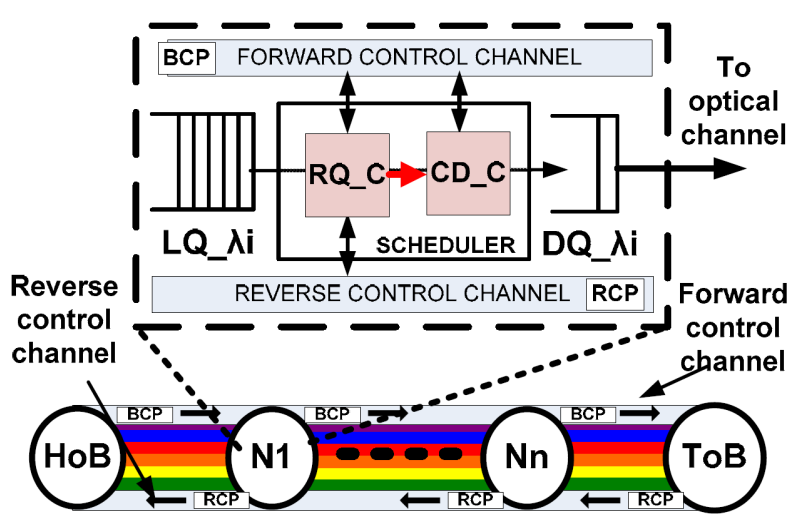

Figure 1. Example of a DAOBS bus.

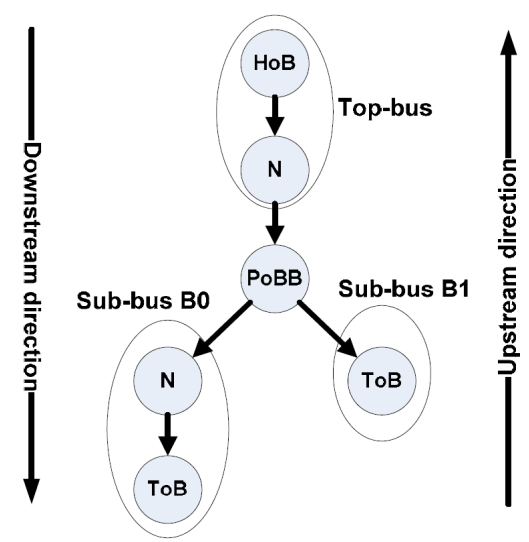

Figure 2. A tree-based topology. 
In this new network configuration, we classify the nodes as:

- Head of Bus (HoB): It is the topmost node of the tree, the root.

- Tail of Bus (ToB): It is the lowest node/s on the tree, or in other words, the tree leafs.

- Point of Bus Branching (PoBB): A node at which the tree is branched into two or more new sub-buses.

- Common node: A node that it is not any of the previous types.

Each node type is responsible for running its own protocol algorithms. For instance, the HoB is responsible for generating at each time slot the $\mathrm{BCP}$; ToBs generate and forward the request packets at every time slot (upon processing the forward $\mathrm{BCP}$ ); and PoBBs are responsible for generating the RCP on the top-bus part of the tree, forward the original BCP towards the destination sub-bus and create a new BCP for the rest of child buses. PoBBs also operate as a barrier between child and parent buses, so that the requests from the child buses are not propagated to the top-bus. In this case, PoBBs keep counting the requests done by their downstream nodes and deliver free/occupied slots according to this number and the occupancy of the upcoming slot from the parent bus. For instance, following Fig. 2, suppose that the current slot is occupied by a slot transmitted from the HoB with destination the ToB of sub-bus B1. In this case, the PoBB forwards the occupied slot (BCP) towards the destination, but as it also manages another sub-bus with the same wavelength, then, depending on the circumstances, it might use the same slot to transmit an own burst to any node of the sub-bus B0, or create a free BCP so that Node $N$ shall have the opportunity to also transmit a burst to its ToB.

An OBS node has one DAOBS entity for every accessible wavelength tree; hence the number of entities is closely related to the number of wavelengths the node can use. Note that due to the tree topology of wavelengths used in the architecture, the wavelength assignment for a certain burst transmission is restricted to wavelengths that permit the burst to reach the destination node without wavelength conversion.

\section{PERFORMANCE EVALUATION}

This section analyzes the performance of the newly proposed DAOBS protocol in front of standard DR. To this end, simulations are conducted on the well-known NSFNET reference network topology, composed of 14 nodes and 21 bidirectional links. In such a scenario, each link carries 16 bidirectional wavelengths at 10 Gbps. Moreover, link lengths are set to the real physical distances.

For the traffic characteristics, bursts depart from each node following a Poisson process with rate $1 / \lambda$. These bursts are uniformly distributed to all the remaining nodes of the network, so that the probability of a burst to be sent to any remainder node in the network remains equal. Moreover, burst sizes follow an exponential distribution with mean $100 \mathrm{kB}$. This leads to an exponentially distributed burst length $L$ with mean $1 / \mu=80 \mu \mathrm{s}$, that is, the time needed to transfer a $100 \mathrm{kB}$ burst onto a $10 \mathrm{Gbps}$ link.

Regarding hardware devices, the BCP processing time and the non-blocking matrix switching time are set to $10 \mu$ s and $5 \mu$ s respectively. As in [6], a Fiber Delay Line (FDL) is placed at each input port of the node, which compensates the processing delay incurred by the BCP at the control unit.

With contention resolution purposes, OBS nodes are equipped with a limited amount of shared wavelength converters (i.e., partial WC in the network), except in DAOBS. As presented in [7], the conversion ratio $R$ is denoted as the number of shared wavelength converters in the node divided by the total number of output wavelength channels. Initially, no wavelength converters are equipped in the OBS nodes (i.e., $R=0.0$ ).

\subsection{Results and discussion}

The results in Fig. 3 validate the performance of DAOBS, which is further compared to conventional unslotted OBS [1]. Two different situations have been considered for the latter: ConvOBS and DeflOBS. ConvOBS assumes no contention resolution in the network, so that the contending burst is inevitably lost if contention exists on the preferred output port. Alternatively, DR is additionally used in DeflOBS to reduce burst losses.

Figure 3a compares the burst loss probability depending on whether DAOBS, ConvOBS or DeflOBS is applied. As shown, the access-admission control in DAOBS results in more than two orders of magnitude burst loss reduction, leading to zero burst loss probability for loads per wavelength lower than 2. In DAOBS, burst losses only occur in ingress nodes, thus avoiding contentions inside the network. Both ConvOBS and DeflOBS become highly affected by the wavelength continuity constraint. Indeed, if $R$ is increased in the network, burst losses for ConvOBS and DeflOBS steadily decrease, showing similar performance than DAOBS for $R$ values around $0.1-0.15$. Note, however, that these metrics are achieved by equipping the network with $72-100$ wavelength converters, thus significantly increasing the network CAPEX to finally obtain a similar performance.

In order to completely assess the performance of DAOBS, Fig. $3 b$ illustrates the mean burst transfer time in ms for DAOBS, ConvOBS and DeflOBS, as a function of the offered load per wavelength in the network. In the figure, as long as $R=0.0$, DeflOBS provides the worst performance. In absence of WC, contentions usually occur on the downstream link. This triggers deflections to alternative (and typically much longer) routes to the destination, drastically impacting on the burst end-to-end delays. In ConvOBS, however, the contending bursts are directly dropped, which makes bursts travelling through longer routes prone to be lost. Hence, towards high loads, being contentions more frequent, the mean burst end-to-end delays are decreased, since only those bursts 
going through shorter routes are finally delivered. Once $R$ is increased in the network, the delays in ConvOBS are also increased as more bursts are delivered. The opposite happens in DeflOBS. As contentions are reduced thanks to the WC, so it is the number of deflections. This is translated into lower delays. Interestingly, DAOBS fits the end-to-end delays of DeflOBS with $R=0.1$, while still providing better performance in terms of burst losses at lower network cost.

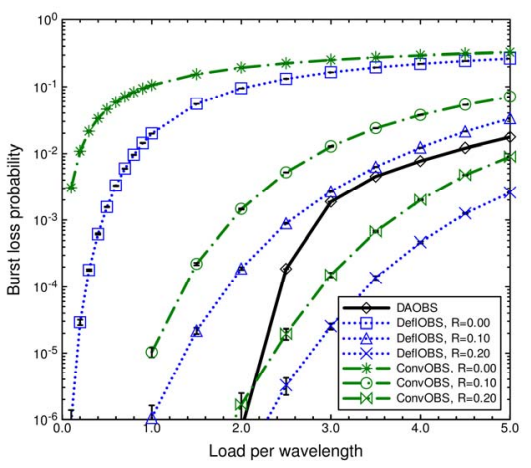

a)

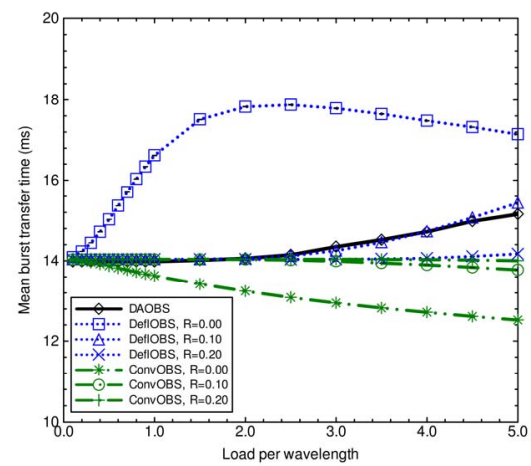

b)

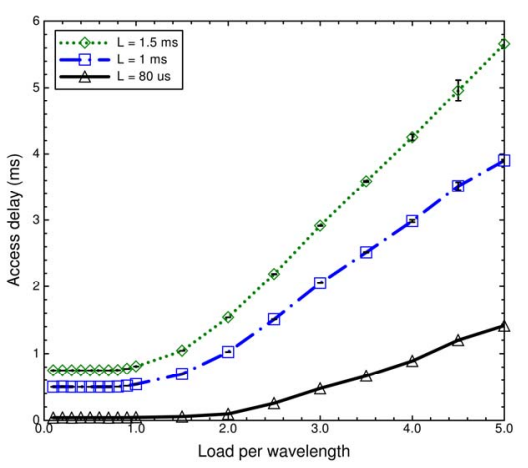

c)

Figure 3: a) Burst loss probability for DAOBS, ConvOBS and DeflOBS; b) mean burst transfer time (ms) for $D A O B S$, ConvOBS and DeflOBS; c) mean access time (ms) for DAOBS.

Heretofore, a quite usual $80 \mu \mathrm{s}$ burst length (100 kB burst size) has been assumed. It shall be pointed out, however, that $L$ has certain influence on the DAOBS access delay. To highlight this, Fig. $3 \mathrm{c}$ shows the DAOBS access delay as a function of the offered load per wavelength for $L$ values of $80 \mu \mathrm{s}, 1 \mathrm{~ms}$ and $1.5 \mathrm{~ms}$. As seen, the access delay in DAOBS depends on both $L$ and the offered load per wavelength. This can be explained as follows. First, the higher the load, the more difficult is to find a free time slot to deliver the burst. Second, the larger the slot size, the longer the waiting time until an upcoming slot becomes available. Nevertheless, if the values are divided by the slot size, we would see that the mean access delay in slots decreases because the slot length approaches the inter-node propagation delay. Note, however, that although the access time is notably increased for $L$ equal to $1 \mathrm{~ms}$ and $1.5 \mathrm{~ms}$, these burst lengths are very large and quite restricted to certain OBS scenarios, such as Grid over OBS.

\section{CONCLUSIONS}

In this paper we have presented a new MAC protocol for Optical Burst Switching specially conceived for network environments without wavelength conversion. The protocol is a useful option in scenarios where minimizing network costs is a requisite or as a mid-term solution whilst other contention resolution schemes using optical enabling technologies are still immature. Simulation results show that in absence of WC, DAOBS outperforms the ConvOBS or DeflOBS regarding burst losses at expenses of slightly increasing the end-to-end delay at high loads. Besides, it gives results comparable to a scenario with a wavelength conversion rate of 0.1 0.15 while still lowering the network budget.

\section{ACKNOWLEDGEMENTS}

This work has been supported by the i2CAT Foundation through the project TRILOGY and the Government of Catalonia and the European Social Funding through a pre-doctoral grant.

\section{REFERENCES}

[1] Y. Chen, C. Qiao, and X. Yu, "Optical burst switching: A new area in optical networking research", IEEE Network, vol. 18, no. 3, pp. 16-23, May 2004.

[2] S. Yao, B. Mukherjee, and S. Dixit, “Advances in photonic packet switching: an overview”, IEEE Communications Magazine, vol. 38, no. 2, pp. 84-94, Feb. 2000.

[3] X. Wang, H. Morikawa, and T. Aoyama, "Burst optical deflection routing protocol for wavelength routing WDM networks", in Proc IEEE/SPIE OptiComm 2000, Dallas, TX, USA, Sept. 2000.

[4] IEEE Standards Board, "Distributed queue dual bus (DQDB) subnetwork of a metropolitan network (MAN) - 802.6", Dec. 1990.

[5] J. Triay and C. Cervelló-Pastor, "Distributed contention avoidance in optical burst-switched ring networks", in Proc. $11^{\text {th }}$ Int. Conf. on Communication Systems (ICCS), Guangzhou, China, Nov. 2008.

[6] M. Klinkowski, D. Careglio, and J. Solé-Pareta, "Comparison of conventional and offset time-emulated optical burst switching", in Proc. $8^{\text {th }}$ Int. Conf. on Transparent Optical Networks (ICTON), pp. 47-50, Nothingham, UK, Jun. 2006.

[7] C.M. Gauger, "Performance of Converter Pools for Contention Resolution in Optical Burst Switching", in Proc. of OptiComm 2002, Jul. 2002. 